\title{
A Design Chart for Long Vacuum Pipes and Shells
}

\author{
K. Krempetz, J. Grimson and P. Kelly \\ Fermi National Accelerator Laboratory \\ P.O. Box 500 \\ Batavia, Illinois 60510
}

July 22, 1986 
TM-1378

July 22, 1986

\section{A DESIGN CHART FOR LONG VACUUM PIPES AND SHELLS}

K. Krempetz, J. Grimson, and P. Kelly

Introduction

This paper presents a design chart to aid designers in the selection of a wall thickness for long cylindrical shells having atmospheric pressure outside the shell and a pressure less than atmospheric inside the shell.

The chart indicates a conservative value for the minimum wall thickness for a given shell diameter and material when the shell is completely evacuated.

Criteria Used for the Chart

Using Fermilab Standard SD-41. the design criteria for the shell shall be a minimum collapsing pressure of 30 psi differential. From Roark and Young. Table 35. Case 19A, for a very long tube of length $\ell$ with free ends, the collapse or critical pressure is given by

$$
\mathrm{P}_{\mathrm{c}}=\frac{1}{4} \frac{\mathrm{E}}{1-\nu^{2}} \frac{\mathrm{T}^{3}}{\mathrm{R}^{3}}
$$

where $\quad E=$ modulus of elasticity

$$
\begin{aligned}
& T=\text { wall thickness } \\
& \nu=\text { Poisson's ratio } \\
& \mathrm{R}=\text { outer radius of the shell } \\
& \mathrm{P}_{\mathrm{c}}=\text { collapsing or critical pressure. }
\end{aligned}
$$

Writing $R$ as $D / 2$, where $D$ is the cylinder's outer diameter, and solving for the wall thickness. T. gives

$$
\mathrm{T}=\mathrm{D}\left(\frac{\left(1-\nu^{2}\right) \mathrm{P}_{\mathrm{c}}}{2 \mathrm{E}}\right)^{1 / 3}
$$

With this equation and the respective material properties for carbon and stainless steel, aluminum and copper, the following curves were plotted:

Curve 1: for carbon steel and stainless steel

$$
\begin{aligned}
& \mathrm{P}=30 \mathrm{psi} \\
& \mathrm{E}^{\mathrm{c}}=30 \times 10^{6} \mathrm{psi} \\
& \nu=0.28
\end{aligned}
$$

Curve 2: for aluminum

$$
\begin{aligned}
& \mathrm{P}=30{ }_{6} \mathrm{psi} \\
& \mathrm{E}^{\mathrm{C}}=10^{\mathrm{psi}} \\
& \nu=0.33
\end{aligned}
$$

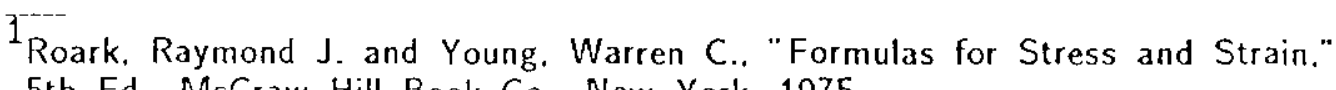

5th Ed.. McGraw Hill Book Co.. New York. 1975. 
Curve 3: for copper

$$
\begin{aligned}
& \mathrm{P}=30 \mathrm{psi} \\
& \mathrm{E}^{\mathrm{C}}=17 \times 10^{6} \mathrm{psi} \\
& \nu=0.33
\end{aligned}
$$

\section{Precautions}

The curves presented in this paper are conservative provided the following precautions are observed:

1) The chart is only applied to $R / T$ ratios greater than 10 .

2) The chart is only applied to very long cylindrical shells where $\ell$ is greater than $4.9 R \sqrt{ } / T$ (for shorter tubes wall thickness can be thinner).

3) The chart is only applied to cylindrical shells with free ends; ends that are not held circular. (For tubes with ends held circular the wall thicknesses can be thinner.)

4) The chart is only applied to a uniform lateral (radial) external pressure and does not incorporate longitudinal (axial) loads.

5) The chart is only applied to perfect cylindrical shapes. A shape is considered to be a perfect cylinder when the maximum difference between the maximum and minimum inside diameters at any cross-section does not exceed one percent of the nominal diameter. See the ASME Boiler and Pressure Vessel Code, Section VIII. Division 1. Paragraph UG-80 for additional information.

\section{Conclusion}

Acceptable wall thicknesses for cylindrical shells of copper, aluminum, carbon or stainless steel subjected to an external pressure differential of 15 psi may easily be obtained. given the outer diameter of the shell. by consulting the presented chart. The thicknesses obtained will have a minimum safety factor of 2 based on the collapsing pressure of the shell. From the data presented it should be apparent that any pipe based on the IPS Standard can withstand 15 psi external pressure (with a safety factor of 2).

The chart presented should not be used if it is absolutely essential to minimize the wall thickness of a shell. There are other approaches. that consider more parameters, that may be used to reliably obtain safe but less conservative values for wall thicknesses. 
MINIMUN WALL THICKNESS FOR LONG VACUUM PIPES

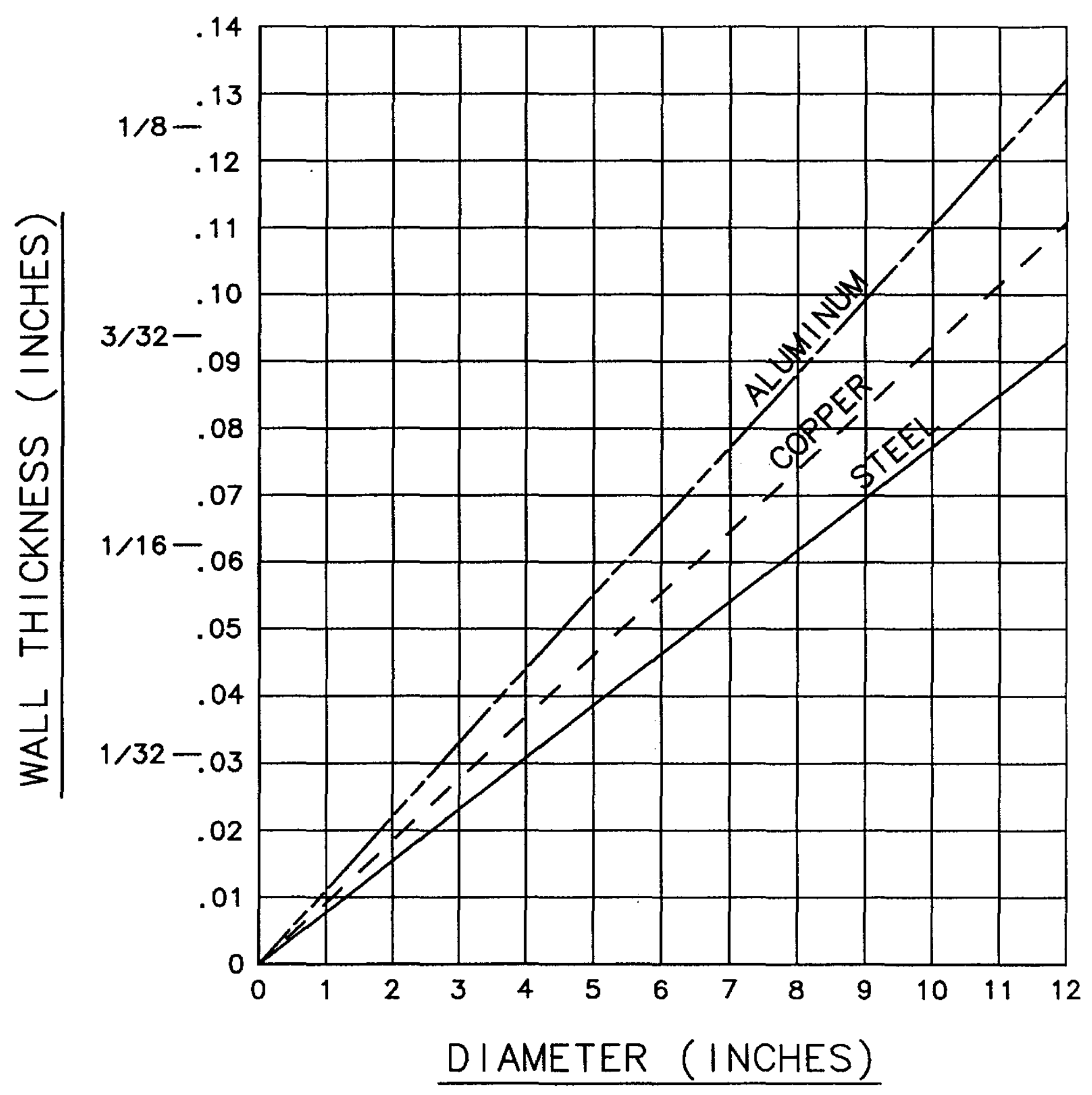

